\title{
ANÁLISE DA EFICÁCIA DE DENTIFRÍCIOS CLAREADORES E SEUS EFEITOS NA SUPERFÍCIE DENTAL: UM ESTUDO IN VITRO
}

\author{
Analysis of the effectiveness of whitening dentifrices \\ and their effects on the dental surface: an in vitro study
}

\author{
DEduardo Monteiro Toschia , (DEliseu Aldrighi Münchow ${ }^{b}$, \\ (DLuísa Weber Mercado ${ }^{a}$, (DRafael Melara ${ }^{b}$, (DSandra Liana Henz ${ }^{a}$
}

\section{RESUMO}

O clareamento dos dentes pode ser realizado de várias maneiras, em consultório, no âmbito caseiro ou com a utilização de produtos OTC (Over-the-Counter). Dentre esses produtos, existem os dentifrícios clareadores que prometem clarear e prevenir o manchamento dentário. Objetivos: (1) Investigar o potencial clareador e abrasivo de diferentes dentifrícios no esmalte; e (2) avaliar o efeito do dentifrício Oral-B 3D White Perfection ${ }^{\circledR}$ (Oral-B) na prevenção do escurecimento. Materiais e Métodos: Amostras de esmalte bovino foram pigmentadas e escovadas por 14 dias com água (controle negativo) ou com diferentes produtos: Colgate Total $12^{\circledR}$ (controle), Oral-B, Sensodyne Branqueador Extra Fresh ${ }^{\circledR}$ (Sensodyne) ou Colgate Luminous White Advanced ${ }^{\circledR}$ (Colgate). Oral-B foi testado antes e após pigmentação. As amostras foram avaliadas quanto à cor (espectrofotômetro) e rugosidade superficial (rugosímetro). Para cálculo de alteração de cor $\left(\Delta \mathrm{DE}_{\text {oo }}\right)$ se utilizou o sistema CIEDE2000. Os dados foram analisados com os testes Kruskal-Wallis, SNK e Mann-Whitney $(\alpha=5 \%)$. Resultados: O controle negativo apresentou a menor $\Delta \mathrm{E}_{\mathrm{oo}}(\mathrm{p}<0,05)$ e o grupo tratado com Sensodyne resultou nos maiores valores $(\mathrm{p}<0,05)$. Quanto à rugosidade superficial, não houve diferença significativa entre os grupos avaliados. O dentifrício Oral-B demonstrou efeito protetivo ao manchamento, resultando em menor alteração de cor $(p=0,002)$. Conclusão: Somente o dentifrício Sensodyne apresentou potencial clareador maior que o dentifrício convencional. A escovação prévia com Oral-B reduziu o manchamento dental, embora não tenha prevenido este totalmente de acontecer. Não foram observadas alterações significativas na rugosidade dos dentes após aplicação dos tratamentos, quando comparado ao controle.

Palavras-chave: Clareamento dental. Esmalte dentário. Dentifrícios. Cor. Abrasão dentária. Pigmentação.

\section{ABSTRACT}

Tooth whitening can be performed at the dental office, at-home or upon the use of OTC (Over-the-Counter) products. Among the latter, there are whitening toothpastes that promise to bleach and to prevent tooth staining. Objectives: (1) To investigate the bleaching and abrasive potential of different whitening dentifrices to enamel; and (2) to evaluate the effect of Oral-B 3D White Perfection ${ }^{\circledR}$ (Oral-B) toothpaste in preventing staining. Materials and Methods: Bovine enamel samples were stained and brushed for 14 days with water (negative control) or with different products: Colgate Total $12{ }^{\circledR}$ (control), Oral-B, Sensodyne Branqueador Extra Fresh ${ }^{\circledR}$ (Sensodyne), and Colgate Luminous White Advanced ${ }^{\circledR}$ (Colgate). Oral-B was also tested before and after staining. The samples were tested by their color (spectrophotometer) and surface roughness (profilometer). Color change $(\Delta \mathrm{DE} \mathrm{oo})$ was calculated using the CIEDE200o color system. The data were analyzed using Kruskal-Wallis, SNK, and Mann-Whitney tests $(\alpha=5 \%)$. Results: The negative control showed the lowest $\Delta \mathrm{E}_{\mathrm{oo}}(\mathrm{p}<0.05)$, whereas the group treated with Sensodyne resulted in the greatest color change $(\mathrm{p}<0.05)$. For surface roughness, there was not any significant difference among the groups. The Oral-B toothpaste showed a protective effect against staining, resulting in the lowest color change $(p=0,002)$. Conclusion: Only Sensodyne toothpaste showed higher whitening potential

âniversidade Federal do Rio Grande do Sul, Faculdade de Odontologia, Laboratório de Bioquímica e Microbiologia, Porto Alegre, RS, Brasil. bUniversidade Federal do Rio Grande do Sul, Faculdade de Odontologia, Dentística, Porto Alegre, RS, Brasil.

Autor de correspondência: Eduardo Monteiro Toschi - E-mail: edu.toschi@hotmail.com

Data de envio: 05/10/2020 | Data de aceite: 09/12/2020 
than the conventional toothpaste. By using Oral-B prior staining reduced significantly the intensity of staining, although without the complete prevention of this event. Significant differences were not observed after treatment concerning roughness in comparison to the control.

Keywords: Tooth bleaching. Dental enamel. Dentifrices. Color. Tooth abrasion. Pigmentation.

\section{INTRODUÇÃO}

Sorrisos claros e saudáveis são características que se refletem não apenas na aparência física de uma pessoa, mas também em sua autoestima, saúde mental e capacidade de se relacionar socialmente, influenciando assim a qualidade de vida ${ }^{1}$. Nos últimos anos, com a tendência crescente pelo desejo de dentes mais brancos e melhor aparência estética, o clareamento dental se tornou uma escolha popular de tratamento entre os pacientes ${ }^{2}$. O clareamento dental é um procedimento conservador, não-invasivo e efetivo para a remoção de manchas intrínsecas e extrínsecas causadoras do escurecimento dental ${ }^{3,4}$.

O clareamento dos dentes pode ser realizado de várias maneiras, seja profissionalmente em consultório, bem como no âmbito caseiro. Nesta última modalidade, o clareamento pode ser monitorado pelo dentista (técnica caseira supervisionada) ou também sem a prescrição e supervisão profissional (técnica caseira não-supervisionada), sendo esta última uma modalidade que se utiliza de produtos Over-the-Counter (OTC). Neste caso, cada pessoa decide pelo produto e a maneira pela qual ele será utilizado ${ }^{5-7}$. De maneira geral, o potencial clareador dos produtos utilizados sob a supervisão profissional é maior e mais efetivo no tratamento de manchas extrínsecas e intrínsecas, ao passo que o potencial clareador dos produtos OTC tende a ser menor, atuando somente sobre manchas extrínsecas.

Existem vários produtos OTC, como os dentifrícios clareadores, fitas clareadoras, enxaguantes clareadores, ou seja, produtos que não necessitam prescrição e que estão disponíveis para aquisição em farmácias, supermercados, ou em lojas virtuais da internet. Entretanto, ainda não se sabe quais são os seus efeitos com o uso continuado e a longo prazo sobre os dentes, mucosas e saúde em geral. Um estudo mostrou que o uso dos produtos OTC produziu alterações topográficas no esmalte, aumentando a sua rugosidade, ranhuras e porosidade ${ }^{8}$. Em outro estudo, o qual comparou o efeito clareador de diferentes produtos OTC (dentifrício clareador, enxaguatório bucal, gel para pintura e um conjunto de fitas clareadoras) ao obtido com a técnica de clareamento caseiro usando-se peróxido de carbamida 10\%, observou-se que quase todos os produtos OTC foram efetivos em promover o clareamento dos dentes, exceto o dentifrício clareador, o qual resultou em efeito similar ao do dentifrício não-clareador controle . Por outro lado, vários trabalhos têm demonstrado que alguns dentifrícios clareadores são eficazes no clareamento dos dentes ${ }^{9-12}$, podendo este efeito acontecer por meio da ação conjunta ou isolada de agentes abrasivos e químicos presentes nos produtos OTC ${ }^{1}$.

Considerando-se a ampla variedade de agentes clareadores presente no mercado, e que, eventualmente, o retoque no clareamento pode ser almejado pelos pacientes, torna-se importante avaliar a possibilidade de se utilizar um dentifrício clareador, o qual é menos potente que os produtos utilizados nas técnicas de consultório ou caseira supervisionada, para a manutenção de um clareamento já realizado. Além disso, alguns dentifrícios clareadores são vendidos com a premissa de atuarem na prevenção do escurecimento/pigmentação dental, como por exemplo o dentifrício Oral-B 3D White Perfection ${ }^{\circledR}$. De fato, são poucos os estudos que tenham se proposto a investigar o efeito destes aspectos no potencial clareador e/ou abrasivo dos dentes. Assim, o presente estudo teve dois objetivos: (1) investigar o potencial clareador e abrasivo de diferentes dentifrícios clareadores; e (2) avaliar o efeito do dentifrício Oral-B 3D White Perfection ${ }^{\circledR}$ na prevenção do escurecimento dental. Para isso, duas hipóteses foram testadas: (1) o potencial clareador e abrasivo dos dentifrícios clareadores 
será maior do que a de um dentifrício convencional (controle); e (2) o dentifrício Oral-B 3D White Perfection ${ }^{\circledR}$ apresentará um efeito protetivo contra a pigmentação dental se aplicado previamente ao processo de pigmentação.

\section{MATERIAIS E MÉTODOS}

Esse estudo in vitro foi realizado no Laboratório de Bioquímica e Microbiologia (LABIM) da Faculdade de Odontologia da Universidade Federal do Rio Grande do Sul (UFRGS), após aprovação pela Comissão de Pesquisa da Faculdade de Odontologia da UFRGS (COMPESQ).

\section{Cálculo amostral}

O cálculo amostral foi realizado usando-se o programa SigmaPlot versão 12 (Systat Software Inc., Chicago, IL, EUA). Dessa forma, o cálculo foi baseado no trabalho de Alshara et $a .^{13}$, considerando-se um desenho comparativo entre cinco grupos independentes, uma diferença média na alteração de cor entre os grupos de 1,8 com um desvio-padrão médio de 1,4, e, ainda, considerando-se um poder de teste de $80 \%$ e significância de $5 \%$. O número de amostras estimado foi de $n=16$. Considerando-se uma perda de $20 \%$, o número amostral final foi de $\mathrm{n}=20$.

\section{Tempos de avaliação}

Foram considerados 3 tempos de avaliação: baseline (To), após o processo de pigmentação (T1) e após o processo de escovação (T2). Considerou-se como desfecho principal a diferença média da alteração de cor dos dentifrícios clareadores em relação à alteração de cor obtida com a aplicação de um dentifrício não clareador. O período utilizado no processo de escovação totalizou 14 dias.

Como controle de qualidade do protocolo, os dentes passaram por análises de inspeção visual, mensuração óptica em espectrofotômetro (VITA Easyshade $\mathrm{V}^{\circledR}$ Bad Säckingen, Baden-Württemberg, Alemanha) e mensuração topográfica em rugosímetro (Mitutoyo SJ-201 ${ }^{\circledR}$, Kawasaki, Kanagawa, Japão) para avaliar possíveis modificações na coloração e estrutura superficial das amostras de esmalte antes, durante e após os procedimentos experimentais.

\section{Preparo dos espécimes}

Dentes incisivos bovinos foram acoplados em uma furadeira de bancada (modelo FB 13, Schulz; Joinville, SC, Brasil) e seccionados sob irrigação contínua com uma serra copo de $10 \mathrm{~mm}$ para se obter espécimes de esmalte dentário de $10 \mathrm{~mm}^{2}$. A ponteira do espectrofotômetro foi posicionada na superfície do esmalte e então feita a medição em cada fase. Todas as medições foram feitas com local, horário, iluminação, fundo e medidor padronizados para que não ocorressem desvios. Após o corte, as peças de esmalte bovino foram incorporadas em uma resina autopolimerizável (Resina acrílica autopolimerizante, Clássico, Meliá Paulista; São Paulo, Brasil) para que apenas as superfícies do esmalte ficassem expostas. As superfícies de esmalte foram alisadas e polidas em uma politriz (modelo APL-04, AROTEC; Cotia, SP, Brasil) utilizando-se lixas com granulação 100, seguido por granulação 600, sob um fluxo constante de água ${ }^{14}$. 


\section{Alocação nos grupos}

Os blocos de esmalte foram divididos em 6 grupos contendo 20 espécimes cada e usando os escores da linha de base, com cada grupo tendo média equivalente de escores da linha de base, possuindo valores de coloração e rugosidade semelhantes entre si e entre os grupos ${ }^{14}$. Os espécimes foram alocados de forma aleatória entre os seguintes grupos, conforme produto e/ou protocolo aplicado: água (controle negativo), Colgate Total $12{ }^{\circledR}$ (controle positivo), Oral-B 3D White Perfection ${ }^{\circledR}$ (Oral-B - com escovação prévia), Oral-B (sem escovação prévia), Sensodyne Branqueador Extra Fresh ${ }^{\circledR}$ (Sensodyne), ou Colgate Luminous White Advanced ${ }^{\circledR}$ (Colgate).

Tabela 1: Informações de fabricante e composição química dos dentifrícios utilizados, com destaque (negrito) para os ingredientes ativos contendo potencial clareador.

\begin{tabular}{|c|c|c|}
\hline Dentifrício (Código) & Fabricante & Composição química \\
\hline $\begin{array}{l}\text { Colgate Total } 12^{\circ} \\
\text { (Controle positivo) }\end{array}$ & $\begin{array}{l}\text { Colgate-Palmolive } \\
\text { Comercial Ltda. } \\
\text { (São Paulo, SP, } \\
\text { Brasil) }\end{array}$ & $\begin{array}{l}\text { Fluoreto de Sódio (1450 ppm de Flúor), Triclosan } \\
\text { 0,3\%, Água, Sorbitol, Sílica Hidratada, Copolímero } \\
\text { PVM, Lauril Sulfato de Sódio, Aroma, Carragenina, } \\
\text { Hidróxido de Sódio, Sacarina Sódica, FD\&C Azul nº } \\
\text { D\&C Amarillo, Dipenteno }\end{array}$ \\
\hline $\begin{array}{l}\text { Oral-B 3D White } \\
\text { Perfection (Oral-B) }\end{array}$ & $\begin{array}{l}\text { Procter \& Gamble } \\
\text { (Seropédica, R], } \\
\text { Brasil) }\end{array}$ & $\begin{array}{l}\text { Glicerina, Sílica Hidratada, Hexametafosfato de } \\
\text { Sódio, Água, PEG-6, Aroma, Fosfato Trissódico, Lauril } \\
\text { Sulfato de Sódio, Carragenina, Cocoamidopropil } \\
\text { Betaína, Mica, Sacarina Sódica, PEG-20M, Goma } \\
\text { Xantam, Dióxido de Titânio, Sucralose, Limoneno, } \\
\text { Pigmento Azul } 15\end{array}$ \\
\hline $\begin{array}{l}\text { Sensodyne } \\
\text { Branqueador Extra } \\
\text { Fresh (Sensodyne) }\end{array}$ & $\begin{array}{l}\text { GlaxoSmithKline } \\
\text { (Rio de Janeiro, R], } \\
\text { Brasil) }\end{array}$ & $\begin{array}{l}\text { Fluoreto de Sódio (1384 ppm de íon/ Íon flúor), } \\
\text { Nitrato de Potássio 5\%, Alumina 1,25\%, Sorbitol, } \\
\text { Água, Sílica Hidratada, Glicerina, Tripolifosfato } \\
\text { Pentassódico, Polietileno glicol, Aroma, Dióxido de } \\
\text { Titânio, Taurato de Metilcocoil de Sódio, Cocoami- } \\
\text { dopropil (1-9), Betaína, Goma Xantam, Hidróxido de } \\
\text { Sódio, Sacarina Sódica }\end{array}$ \\
\hline $\begin{array}{l}\text { Colgate Luminous } \\
\text { White Advanced } \\
\text { (Colgate) }\end{array}$ & $\begin{array}{l}\text { Colgate-Palmolive } \\
\text { Comercial Ltda. } \\
\text { (São Paulo, SP, } \\
\text { Brasil) }\end{array}$ & $\begin{array}{l}\text { Propileno Glicol, Pirofosfato de Cálcio, Peróxido de } \\
\text { Hidrogênio 2\%, Copolímero 66, PEG-12, Glicerina, } \\
\text { Aroma, Lauril Sulfato de Sódio, Sílica, PVP, Pirofos- } \\
\text { fato Tetrassódico, Sacarina Sódica, Monofluorfosfato } \\
\text { de Sódio o,76\%, Pirofosfato Dissódico, Sucralose, } \\
\text { BHT, Eugenol }\end{array}$ \\
\hline
\end{tabular}

PEG: polietilenoglicol; PVP: polivinilpirrolidona; BHT: hidroxitolueno butilado.

\section{Protocolo de pigmentação}

A preparação e pigmentação dos dentes foram realizadas conforme o método de Stookey et al. ${ }^{14}$. O processo de pigmentação foi feito através da imersão contínua e alternada entre o caldo de coloração e a secagem ao ar. Os espécimes foram mergulhados na solução por $24 \mathrm{~h}$ e retirados após o período para secagem ao ar por $1 \mathrm{~h}$ e repetido o processo por 14 dias. O caldo de coloração consistiu em uma solução com 2,7 g de café solúvel moído (Café do Mercado ${ }^{\circledR}$ ), 
2,7 g de chá preto instantâneo (Twinings ${ }^{\circledR}$ ), 2,0 g de vinho tinto (Salton ${ }^{\circledR}$ ), 2,0 g de molho de soja (Sakura ${ }^{\circledR}$ ) misturados com $50 \mathrm{~mL}$ de água deionizada. Os espécimes foram colocados em uma estufa a $37^{\circ} \mathrm{C}$ e a pigmentação foi realizada por um processo alternando ar e solução. Após o período de coloração por 14 dias, percebeu-se a criação de filmes/revestimentos na superfície dos dentes. Após a pigmentação, os blocos de esmalte corados foram enxaguados com água deionizada, deixados para secar ao ar por 10 min e mantidos sob refrigeração por 7 dias. Após, os espécimes foram lavados em água deionizada e passaram pelo processo de escovação.

\section{Protocolo de escovação}

Após o processo de coloração, os dentes foram escovados de forma padronizada, por 3 vezes ao dia, durante 14 dias, com cada escovação possuindo 20 movimentos de ida-e-volta, totalizando em cada dente 900 movimentos de escovação (golpes duplos). Apenas um grupo teve o mesmo processo de escovação antes da pigmentação com o intuito de ser observado os resultados de proteção contra a coloração do caldo, como é prometido pelo dentifrício Oral-B. Os dentifrícios foram utilizados como uma pasta consistindo de $25 \mathrm{~g}$ de dentifrício misturados com $40 \mathrm{ml}$ de água deionizada ${ }^{14}$. A diferença de cor entre T1 e T2 foi considerada representando a capacidade do material de remover as pigmentações, enquanto que a diferença de cor entre To e T1 foi considerada para o grupo Oral-B (com escovação prévia) como a capacidade do material teste de evitar a pigmentação.

\section{Análise de cor}

As medidas de cor foram realizadas antes da pigmentação, logo após o processo de pigmentação e após o processo de escovação. Cada espécime foi aferido 3 vezes consecutivas em cada etapa para ser feita uma média de aferição. A diferença entre os escores pré-escovação (após pigmentação) e pós-escovação foi considerada representando a capacidade do material de teste para remover ou evitar as pigmentações.

A alteração de cor $\left(\Delta \mathrm{E}_{00}\right)$ obtida após os processos de pigmentação e escovação foi calculada pelo método CIEDE2000 ${ }^{15}$, utilizando-se a seguinte fórmula:

$$
\Delta \mathrm{E}_{\text {oo }}=\left[\left(\Delta \mathrm{L}^{\prime} / \mathrm{K}_{\mathrm{L}} \mathrm{S}_{\mathrm{L}}\right)^{2}+\left(\Delta \mathrm{C}^{\prime} / \mathrm{K}_{\mathrm{C}} \mathrm{S}_{\mathrm{C}}\right)^{2}+\left(\Delta \mathrm{H}^{\prime} / \mathrm{K}_{\mathrm{H}} \mathrm{S}_{\mathrm{H}}\right)^{2}+\mathrm{R}_{\mathrm{T}}\left(\Delta \mathrm{C}^{\prime} / \mathrm{K}_{\mathrm{C}} \mathrm{S}_{\mathrm{C}}\right)\left(\Delta \mathrm{H}^{\prime} / \mathrm{K}_{\mathrm{H}} \mathrm{S}_{\mathrm{H}}\right)\right]^{1 / 2}
$$

onde $\Delta \mathrm{L}, \Delta \mathrm{C}^{\prime}$ e $\Delta \mathrm{H}^{\prime}$ representam, respectivamente, as diferenças de luminosidade, cor e intensidade entre duas medidas em comparação (por exemplo, valores de baseline $\mathrm{x}$ após pigmentação ou valores obtidos após pigmentação x escovação). $\mathrm{R}_{\mathrm{T}}$ é uma função rotacional que quantifica as diferenças de cor e intensidade na região azul, melhorando a qualidade equacional do cálculo de alteração de cor. As funções $S_{L}, S_{C}$ e $S_{H}$ ajustam a variação de cor total em relação ao grupo tratado L', a' e b’. Já os fatores paramétricos $\mathrm{K}_{\mathrm{L}}, \mathrm{K}_{\mathrm{C}}$ e $\mathrm{K}_{\mathrm{H}}$ são correções realizadas conforme as condições experimentais. Os valores referência para a percepção de alteração de cor e para a aceitabilidade clínica da alteração de cor foram estabelecidos, respectivamente, em $\Delta \mathrm{E}_{00}>0,8$ e $\Delta \mathrm{E}_{00}>1,8^{16}$.

\section{Análise da rugosidade}

Em relação aos danos que poderiam surgir na estrutura do esmalte devido ao uso dos dentifrícios clareadores, os dentes bovinos tiveram sua rugosidade aferida através do rugosímetro. Para medições da rugosidade superficial, foi utilizado o rugosímetro, o qual com uma agulha possuindo um diamante muito fino em sua ponta foi conduzida a uma velocidade constante de $0,5 \mathrm{~mm} / \mathrm{s}$ ao longo de uma distância de 2,5 mm. Três leituras foram feitas em locais diferentes na superfície da amostra para se obter a rugosidade média (Ra) ${ }^{17}$. 
As amostras foram avaliadas antes do processo de pigmentação e após o processo de escovação. Cada espécime foi aferido 3 vezes consecutivas em cada etapa e o valor de rugosidade foi calculado como a média das 3 medições, em $\mu \mathrm{m}$. Os valores de rugosidade se baseiam no parâmetro de avaliação da rugosidade Ra em que se realiza a média aritmética dos valores absolutos das ordenadas de afastamento (yi), dos pontos do perfil de rugosidade em relação à linha média, dentro do percurso de medição $(\mathrm{lm})$. Para o cálculo da alteração da rugosidade superficial $(\triangle \mathrm{Ra})$, o valor de Ra inicial foi subtraído do valor de Ra final.

\section{Análise estatística}

Todas as análises estatísticas conduzidas neste estudo foram realizadas utilizando-se o programa SigmaPlot versão 12, já descrito anteriormente. Os dados foram analisados quanto à normalidade pelo teste de Shapiro-Wilk, os quais não cumpriram uma distribuição paramétrica. Os dados relacionados ao potencial clareador e abrasivo dos diferentes dentifrícios foram analisados com Kruskal-Wallis seguido do teste complementar de Student-Newman-Keuls. Por sua vez, os dados relacionados ao efeito do dentifrício Oral-B na prevenção da pigmentação foram analisados com o teste de Mann-Whitney. O nível de significância foi de $5 \%$ para todas as análises realizadas.

\section{RESULTADOS}

A Tabela 2 mostra os resultados de alteração de $\operatorname{cor}\left(\Delta \mathrm{E}_{00}\right)$ e de alteração da rugosidade superficial $(\triangle \mathrm{Ra})$ obtida após o protocolo de escovação com os diferentes dentifrícios. Todos os grupos avaliados resultaram em valores de $\Delta \mathrm{E}_{\text {oo }}$ maiores do que 1,8 , ou seja, acima do limiar de aceitabilidade clínica de mudança de cor. Contudo, o controle negativo apresentou a menor mudança de cor do estudo, a qual foi estatisticamente menor do que a observada com os grupos tratados com o dentifrício controle e com os dentifrícios clareadores Oral-B e Colgate $(\mathrm{p}<0,05)$. Por sua vez, o grupo tratado com Sensodyne resultou na maior alteração de cor do estudo, sendo esta significativamente superior quando comparada a todos os demais grupos $(\mathrm{p}<0,05)$.

Tabela 2: Valores de mediana (mínimo e máximo) da alteração de cor $\left(\Delta \mathrm{DE}_{\mathrm{oo}}\right.$ ) e alteração da rugosidade superficial $(\triangle \mathrm{Ra}$ ) após escovação com diferentes tipos de dentifrícios.

\begin{tabular}{lll}
\hline Grupo & $\boldsymbol{\Delta D E}_{\mathrm{oO}}$ & $\boldsymbol{\Delta R a}(\boldsymbol{\mu m})$ \\
\hline Controle negativo & $2,6(0,9-4,5)^{\mathrm{C}}$ & $0,117(-0,160-0,993)$ \\
Dentifrício controle & $5,8(0,7-14,7)^{\mathrm{B}}$ & $-0,190(-2,637-1,316)$ \\
Oral-B & $4,9(1,6-18,6)^{\mathrm{B}}$ & $0,023(-0,810-3,297)$ \\
Sensodyne & $7,9(2,4-17,2)^{\mathrm{A}}$ & $0,223(-0,330-0,697)$ \\
Colgate & $3,9(2,2-9,9)^{\mathrm{B}}$ & $0,233(-0,683-3,940)$ \\
\hline
\end{tabular}

Letras distintas indicam diferenças estatisticamente significantes entre os grupos ( $p<0,05$; Kruskal-Wallis e Student-Newman-Keuls). 
A Figura 1 mostra a modificação dos parâmetros de cor L*, a* e b* para cada grupo e períodos de tempo investigados no estudo, onde se percebe que o processo de pigmentação contribuiu para a redução da luminosidade das amostras, bem como para o aumento dos tons avermelhado (eixo $a^{*}$ ) e amarelado (eixo b*) das mesmas. Após o processo de escovação, os parâmetros de cor L*, a* e b* foram modificados no sentido de recuperação dos valores originais, embora esta recuperação não tenha sido completa. Apenas o parâmetro de cor b* retornou a valores mais próximos do baseline, com tendência à redução do tom amarelado.

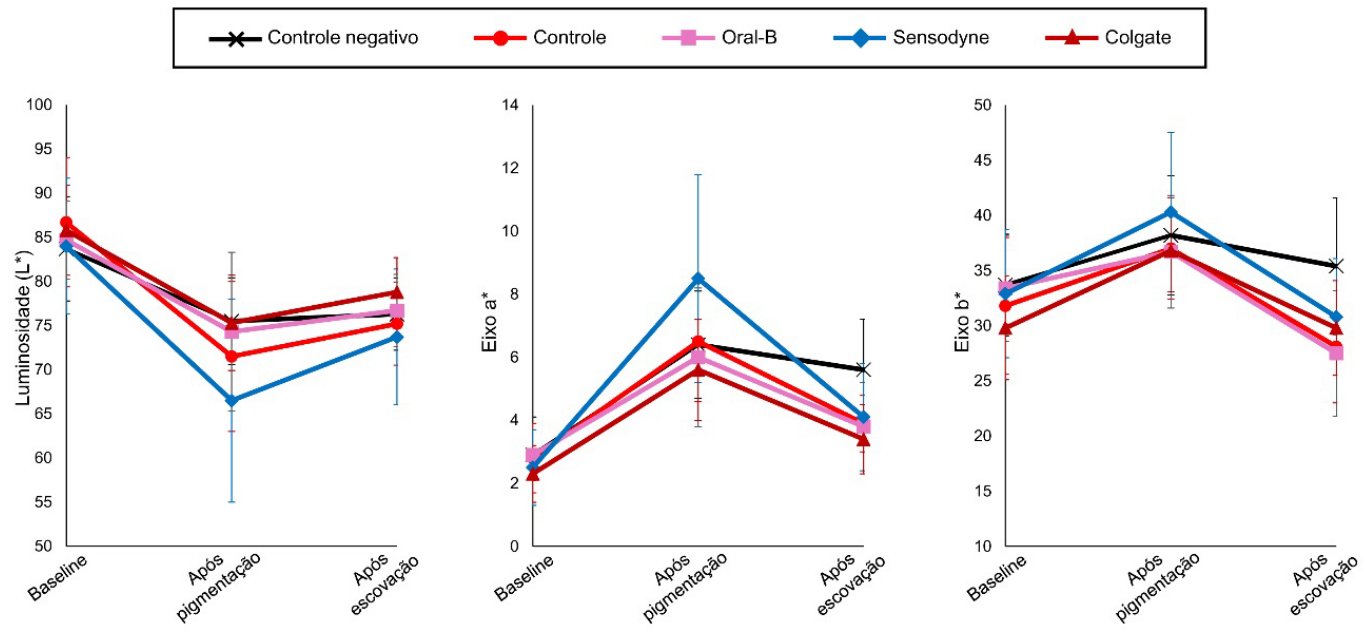

Figura 1: Gráficos demonstrando a variação dos parâmetros de cor L*, a* e b* para cada grupo investigado no estudo e após diferentes períodos de tempo: baseline (antes da pigmentação), após pigmentação e após escovação.

Quanto à alteração da rugosidade superficial, todos os grupos deste estudo apresentaram valores estatisticamente semelhantes entre si ( $p>0,05)$. Porém, conforme apresentado na Figura 2, enquanto a utilização do dentifrício controle resultou em superfícies 16,7\% mais lisas após o processo de escovação, as amostras tratadas com os dentifrícios clareadores tornaram-se $46 \%$ a $~ 77 \%$ mais rugosas após a escovação. O grupo controle negativo também apresentou um aumento ( $32 \%)$ nos valores da sua textura superficial.

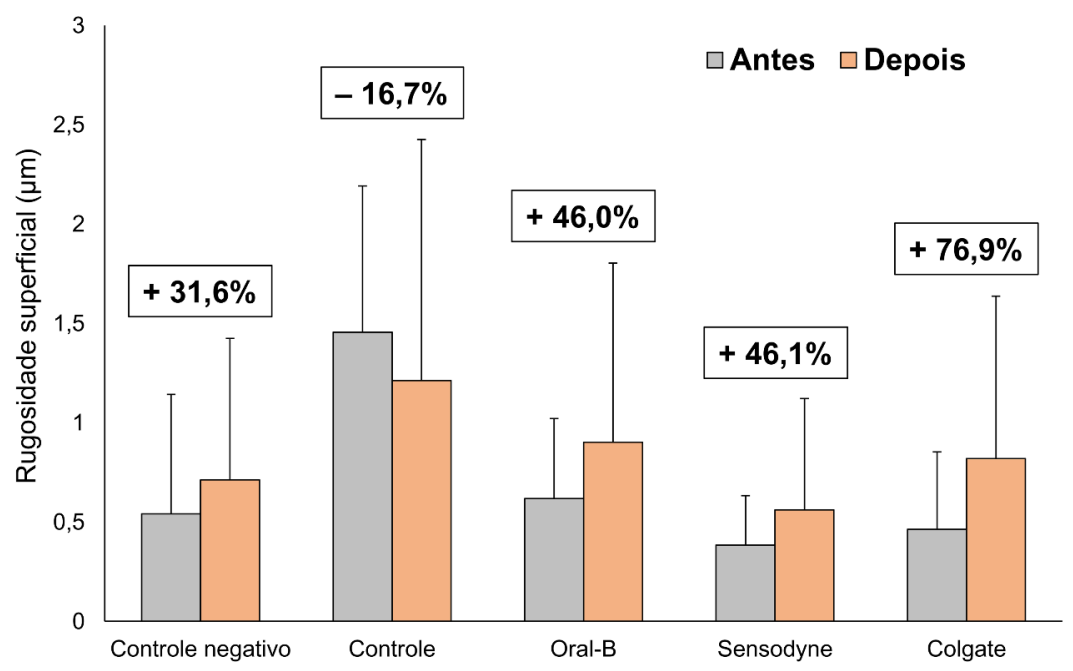

Figura 2: Gráfico demonstrando a variação da rugosidade superficial (aumento ou redução percentual) obtida após a aplicação dos diferentes dentifrícios. 
Relativo ao efeito preventivo à pigmentação por parte do dentifrício Oral-B, os resultados da alteração de cor $\left(\Delta \mathrm{DE}_{00}\right)$ obtidos após pigmentação e clareamento estão demonstrados na Tabela 3. A utilização do referido dentifrício antes do processo de pigmentação produziu valores de $\triangle \mathrm{DE}_{\text {oo }}$ significativamente menores do que aqueles obtidos quando da não escovação prévia com o produto $(\mathrm{p}=0,002)$. Porém, este efeito protetivo não teve influência após o processo de clareamento realizado, onde os valores de $\Delta \mathrm{DE}_{\text {oo }}$ foram semelhantes entre si $(\mathrm{p}=0,516)$, independente da escovação preventiva com o dentifrício.

Tabela 3: Valores de mediana (mínimo e máximo) da alteração de cor $\left(\triangle \mathrm{DE} \mathrm{oo}_{\mathrm{o}}\right.$ ) obtida após pigmentação e clareamento, conforme escovação preventiva ou não das amostras dentais usando-se o dentifrício Oral-B 3D White Perfection.

\begin{tabular}{llc}
\hline \multirow{2}{*}{$\begin{array}{l}\text { Escovação preventiva } \\
\text { antes da pigmentação }\end{array}$} & \multicolumn{2}{c}{ Alteração de cor $(\Delta$ DEoo) } \\
\cline { 2 - 3 } & Após pigmentação & Após clareamento \\
\hline $\operatorname{Sim}$ & $5,0(2,9-10,6)^{\mathrm{B}}$ & $4,2(2,8-6,3)^{\mathrm{A}}$ \\
Não & $8,3(2,1-34,3)^{\mathrm{A}}$ & $4,0(0,7-18,6)^{\mathrm{A}}$ \\
\hline
\end{tabular}

Letras distintas indicam diferenças estatisticamente significantes entre os grupos em cada período avaliado ( $p<0,05$; Mann-Whitney).

\section{Discussão}

O presente estudo mostrou que os dentifrícios utilizados apresentaram potencial clareador nas condições experimentais, pois todos os grupos mostraram alteração de cor significativamente diferente em relação ao controle negativo. Os dentifrícios utilizados apresentam mecanismos variados de clareamento, envolvendo ação química e/ou mecânica para a remoção das manchas extrínsecas. O mecanismo de ação esperado para o peróxido de hidrogênio, presente no dentifrício clareador Colgate, é a reação de oxirredução com as moléculas orgânicas que causam manchamento superficial dos dentes, gerando-se assim moléculas menores, e, com isso, um efeito óptico de dentes mais claros/luminosos ${ }^{18}$. A alteração de cor obtida com os demais dentifrícios pode ser atribuída ao efeito abrasivo das substâncias que os constituem, permitindo a remoção do biofilme e dos cromóforos aderidos às superfícies dos dentes ${ }^{11}$ ou ainda devido a algum efeito químico na prevenção da adsorção das moléculas de pigmento ${ }^{1}$. A presença da sílica hidratada na composição do dentifrício controle pode explicar o efeito clareador visualizado neste grupo experimental, já que a mesma apresenta uma melhor capacidade de remoção de manchas comparada aos outros abrasivos observados nos demais dentifrícios testados ${ }^{9}$. Da mesma forma, a presença de alumina na composição do Sensodyne pode ter contribuído para melhor eficácia clareadora comparada aos demais dentifrícios testados ${ }^{19}$, já que a alumina é um agente mais duro/abrasivo do que os ingredientes encontrados na formulação dos demais dentifrícios.

A presença de abrasivos na composição dos dentifrícios pode gerar tanto um efeito clareador com a remoção de manchas, como também um efeito danoso na superfície do dente, conforme a capacidade abrasiva das substâncias utilizadas ${ }^{19}$. No presente trabalho, a mensuração da rugosidade considerou que a composição dos dentifrícios clareadores possui uma maior carga de ingredientes abrasivos, conferindo assim um certo polimento da estrutura dental e consequentemente a eliminação das manchas extrínsecas presentes no esmalte dos dentes ${ }^{8}$. Contudo, foi observada uma tendência de aumento na rugosidade superficial, exceto para o grupo do dentifrício controle, sugerindo-se assim um potencial abrasivo dos 
dentifrícios clareadores. Vale salientar que não houve diferença significativa entre os grupos e o controle negativo em termos de rugosidade superficial. Mesmo assim, no grupo controle, houve uma diminuição aparente da rugosidade após o processo de escovação, o que poderia ser um efeito concomitante da sílica em promover a limpeza e também o polimento da superfície de esmalte. Apesar disso, os presentes resultados devem ser interpretados com parcimônia, já que no próprio período de baseline, os valores foram mais elevados no grupo controle e portanto diferentes dos demais grupos. Além disso, estudos que avaliaram o uso de dentifrícios contendo sílica têm demonstrado aumento na rugosidade superficial devido às suas propriedades abrasivas ${ }^{20,21}$.

Os resultados de alteração de cor observados neste estudo apresentam valores clinicamente relevantes com $\triangle \mathrm{DE}_{00}$ maior que 1,8(16). É possível verificar que o controle negativo também apresentou alteração da cor acima deste limiar de percepção clínica para alteração de cor, o que pode ser explicado pelo efeito mecânico da escovação na remoção dos pigmentos, reduzindo o manchamento dental de maneira clinicamente significativa. Mesmo assim, a ação abrasiva e detergente dos componentes dos dentifrícios demonstrou maior eficácia na remoção das manchas, promovendo assim melhor clareamento dental. Considerando-se a primeira hipótese do estudo de que o potencial clareador e abrasivo dos dentifrícios clareadores seria maior do que a de um dentifrício não-clareador convencional (controle), a mesma deve ser rejeitada parcialmente, uma vez que somente o dentifrício Sensodyne apresentou potencial clareador maior que o dentifrício convencional. Neste caso em específico, torna-se importante salientar o fato de que os espécimes tratados com o dentifrício Sensodyne resultaram na maior variabilidade de dados relativos aos parâmetros L*, a* e b*, como demonstrado na Figura 1, independentemente do período investigado, o que pode ter então contribuído para a maior alteração de cor visualizada para este grupo após o processo de escovação, sendo essa uma limitação do estudo.

Com a análise de $\Delta \mathrm{DE}_{\mathrm{o} 0}$ comparando os grupos com escovação prévia e sem escovação prévia entre o baseline e após o processo de pigmentação, foi observado uma menor alteração de cor para o grupo em que se aplicou a escovação prévia. Conforme os resultados apresentados na Tabela 3, é possível verificar que houve um efeito protetor à pigmentação, embora não preventivo, por parte do dentifrício Oral-B, aceitando a segunda hipótese do estudo. De fato, sugere-se que o efeito protetor do dentifrício pode ser atribuído à presença e à ação do hexametafosfato de sódio como ingrediente ativo do dentifrício Oral-B, o qual potencializa a redução da adsorção de cromógenos na superfície do dente ${ }^{1}$.

No presente estudo, o processo de escovação ocorreu com o uso de uma escova padronizada entre os grupos com os movimentos definidos e repetidos de forma igual, no intuito de mimetizar a escovação realizada habitualmente. Assim, pode-se esperar que com o uso prolongado dos dentifrícios aqui testados seja possível verificar algum desgaste ou aumento significativo da rugosidade do esmalte devido às diferentes composições dos diversos produtos. Posto isso, são necessários mais estudos que permitam um maior tempo de uso dos dentifrícios a fim de verificar possíveis alterações na estrutura dental, assim como estudos clínicos controlados para confirmar a eficácia dos dentifrícios clareadores.

\section{LIMITAÇÕES DO ESTUDO}

Por se tratar de um estudo in vitro o mesmo apresenta as limitações inerentes a esse tipo de estudo, uma vez que não mimetiza a condição bucal. Além disso, não é possível avaliar a interação dos dentifrícios clareadores com a película adquirida e a saliva que podem influenciar ou modificar a ação dos mesmos. 


\section{CONCLUSÃo}

Somente o dentifrício Sensodyne apresentou potencial clareador maior que o dentifrício convencional. A escovação prévia com o dentifrício Oral-B 3D White Perfection ${ }^{\circledR}$ reduziu a pigmentação dental, embora sem ação preventiva. Não foram observadas alterações significativas na rugosidade dos dentes após aplicação dos tratamentos com os diferentes dentifrícios, quando comparado ao dentifrício controle não clareador.

\section{CONFLITO DE INTERESSES}

Os autores declaram não haver conflito de interesses.

\section{REFERÊNCIAS}

1. Joiner A. Whitening toothpastes: a review of the literature. J Dent. 2010;38 Suppl 2:e17-24.

2. Chen H-P, Chang C-H, Liu J-K, Chuang S-F, Yang J-Y. Effect of fluoride containing bleaching agents on enamel surface properties. J Dent. 2008 Sep;36(9):718-25.

3. Braun A, Jepsen S, Krause F. Spectrophotometric and visual evaluation of vital tooth bleaching employing different carbamide peroxide concentrations. Dent Mater. 2007 Fev;23(2):165-9.

4. Joiner A. The bleaching of teeth: a review of the literature. J Dent. 2006 Aug;34(7):412-9.

5. Alqahtani MQ. Tooth-bleaching procedures and their controversial effects: a literature review. Saudi Dent ]. 2014 Apr;26(2):33-46.

6. Kwon SR, Wertz PW. Review of the mechanism of tooth whitening: the mechanism of tooth whitening. J Esthet Restor Dent. 2015 Sep;27(5):240-57.

7. Kwon SR, Kurti SR, Oyoyo U, Li Y. Effect of various tooth whitening modalities on microhardness, surface roughness and surface morphology of the enamel. Odontology. 2015 Sep;103(3):274-9.

8. Omar F, Ab-Ghani Z, Rahman NA, Halim MS. Nonprescription bleaching versus home bleaching with professional prescriptions: which one is safer? A comprehensive review of color changes and their side effects on human enamel. Eur] Dent. 2019 Oct;13(4):589-98.

9. Karadas M, Duymus ZY. In Vitro evaluation of the efficacy of different over-the-counter products on tooth whitening. Braz Dent ]. 2015 Aug;26(4):373-7.

10. Casado BCS, Moraes SLD, Souza GFM, Guerra CMF, Souto-Maior]R, Lemos CAA, et al. Efficacy of dental bleaching with whitening dentifrices: a systematic review. Int ] Dent. 2018 Oct;2018:7868531.

11. Vaz VTP, Jubilato DP, Oliveira MRM de, Bortolatto JF, Floros MC, Dantas AAR, et al. Whitening toothpaste containing activated charcoal, blue covarine, hydrogen peroxide or microbeads: which one is the most effective? ] Appl Oral Sci. 2019;27:e20180051.

12. Devila A, Lasta R, Zanella L, Agnol MD, Rodrigues-Junior S. Efficacy and adverse effects of whitening dentifrices compared with other products: a systematic review and meta-analysis. Oper Dent. 2020 Mar;45(2):E77-90.

13. Alshara S, Lippert F, Eckert G], Hara AT. Effectiveness and mode of action of whitening dentifrices on enamel extrinsic stains. Clin Oral Investig. 2014 Mar;18(2):563-9.

14. Stookey GK, Burkhard TA, Schemehorn BR. In vitro removal of stain with dentifrices.] J Dent Res. 1982 Nov;61(11):1236-9.

15. Sharma G, Wu W, Dalal EN. The CIEDE200o color-difference formula: Implementation notes, supplementary test data, and mathematical observations. Color Res Appl. 2005 Fev;30(1):21-30.

16. Paravina RD, Ghinea R, Herrera L], Bona AD, Igiel C, Linninger M, et al. Color difference thresholds in dentistry: color difference thresholds. ] Esthet Restor Dent. 2015 Mar;27 Suppl 1:S1-9.

17. Rodrigues SB, Leitune VCB, Hashizume LN, Samuel SMW, Collares FM. Polímero de MMA para base de dentadura com a adição de subnitrato de bismuto. Rev Fac Odontol - UPF. 2014 maio-ago;19(2):166-71.

18. Rodríguez-Martínez ], Valiente M, Sánchez-Martín M. Tooth whitening: from the established treatments to novel approaches to prevent side effects. J Esthet Restor Dent. 2019 Sep;31(5):431-40. 
19. Subramanian S, Appukuttan D, Tadepalli A, Gnana PPS, Victor D]. The role of abrasives in dentifrices. ] Pharm Sci. 2017:9(2):221-4.

20. Hilgenberg SP, Pinto SCS, Farago PV, Santos FA, Wambier DS. Physical-chemical characteristics of whitening toothpaste and evaluation of its effects on enamel roughness. Braz Oral Res. 2011 Jul;25(4):288-94.

21. Rahardjo A, Gracia E, Riska G, Adiatman M, Maharani D. Potential Side Effects of Whitening Toothpaste on Enamel Roughness and Micro Hardness. Int ] Clin Prev Dent 2015;11(4):239-42. 